\title{
Verifying single-mode nonclassicality beyond negativity in phase space
}

\author{
Jiyong Park $\odot,{ }^{1, *}$ Jaehak Lee $\odot,{ }^{2}$ and Hyunchul Nha $\odot^{3, \dagger}$ \\ ${ }^{1}$ School of Basic Sciences, Hanbat National University, Daejeon 34158, Korea \\ ${ }^{2}$ School of Computational Sciences, Korea Institute for Advanced Study, Seoul 02455, Korea \\ ${ }^{3}$ Department of Physics, Texas A\&M University at Qatar, Education City, P.O. Box 23874, Doha, Qatar
}

(Received 22 June 2020; accepted 3 November 2021; published 15 November 2021)

\begin{abstract}
While negativity in phase space is a well-known signature of nonclassicality, a wide variety of nonclassical states require their characterization beyond negativity. We establish a framework of nonclassicality in phase space that addresses nonclassical states comprehensively with a direct experimental evidence. This includes the negativity of phase-space distribution as a special case and further analyzes quantum states with positive distributions effectively. We prove that it detects all nonclassical Gaussian states and all non-Gaussian states of arbitrary dimension remarkably by examining three phase-space points only. Our formalism also provides an experimentally accessible lower bound for a nonclassicality measure based on trace distance. Importantly, this foundational approach can be further adapted to constitute practical tests in two directions looking into particle and wave nature of bosonic systems, via an array of nonideal on-off detectors and coarse-grained homodyne measurement, respectively. All these tests are practically powerful in characterizing nonclasssical states reliably against noise, making a versatile tool for a broad range of quantum systems in quantum technologies.
\end{abstract}

DOI: 10.1103/PhysRevResearch.3.043116

\section{INTRODUCTION}

Describing a quantum state of light or matter in phase space, e.g., Wigner function [1], is profoundly important to study quantum dynamics. It is a crucial tool to delineate the boundary between classical and quantum physics widely used in quantum optics [2], continuous variable $(\mathrm{CV})$ quantum informatics [3,4], and other fields of quantum science [5]. As a classical phase-space distribution takes non-negative values like a probability distribution, a negativity emerging in quantum distribution is regarded as a signature of nonclassicality. However, negativity is just one aspect of multifaceted nonclassicality characterizing only a subset of nonclassical states. There exist quantum states with positive distributions that can nevertheless be classified as nonclassical, e.g., a squeezed state of light that is a key resource for $\mathrm{CV}$ quantum informatics [4] and single photons under a high-loss channel that are elementary information carriers for quantum informatics $[6,7]$.

Nonclassical states are essential resources broadly for quantum informatics generating entangled states [8-12], providing advantage for quantum metrology [13-15] and quantum computation $[16,17]$, etc.. A recent resource theory identified all quantum non-Gaussian states, even with positive

\footnotetext{
*jiyong.park@hanbat.ac.kr

†hyunchul.nha@qatar.tamu.edu
}

Published by the American Physical Society under the terms of the Creative Commons Attribution 4.0 International license. Further distribution of this work must maintain attribution to the author(s) and the published article's title, journal citation, and DOI.
Wigner functions, as a resource for quantum tasks, e.g., subchannel discrimination [18], which was further generalized to all CV nonclassical states [19]. It is thus crucial to establish a framework that can widely analyze nonclassical states beyond negativity. Specific properties were often used to characterize nonclassical states such as squeezing and photon-number statistics (sub-Poissonian) [20-23] extended also to multimode cases [24,25]. Distillation of nonclassicality can also be used to verify the nonclassicality of an initial state, however, requiring multiple copies of the same nonclasscial state and postselection [26-28]. More broadly, a quantum state tomography may be used to obtain complete information on a state thereby confirming nonclassicality [29]. However, it requires extensive measurements for sufficient data, and more seriously, an optimization process to find a physical state closest to obtained data. The data itself does not directly represent a legitimate quantum state rendering its significance weaker. It is necessary to characterize nonclassicality by examining phase space in a faithful and resource-efficient way.

Adhering to negativity as a nonclassical feature, some works proposed to display negativity by modifying phasespace distributions, e.g., a regularized $P$ function under filtering process $[30,31]$. Other distributions closely related to the so-called $s$-parametrized functions [2] were also studied in view of photon statistics from on-off detectors [32,33]. Phase-space inequalities were also obtained by combining different $s$-parametrized functions useful to some extent [34]. Nevertheless, it is worth asking if the original Wigner function contains substantial information on nonclassicality beyond negativity. In this respect, Banaszek and Wódkiewicz proposed a Bell test examining four phase-space points to manifest nonlocality of two-mode states with positive Wigner functions [35], which were extended to generalized 
quasiprobability distributions [36] and genuine multipartite nonlocality [37-39]. The works in [40,41] demonstrated Bell-like tests also for single-mode nonclassicality and quantum non-Gaussianity. While conceptually remarkable and practically useful, these methods do not address a broad range of nonclassical states, e.g., squeezed states with purity $<0.86$ are out of reach.

In this article, we propose a hierarchy of nonclassicality criteria in phase space that yields an efficient and broadly applicable test for CV systems. Our formalism addresses the Wigner function at $\frac{n(n+1)}{2}$ phase-space points progressively $(n=1,2, \ldots)$. It includes the negativity of Wigner function at $n=1$. Remarkably, it can detect all nonclassical Gaussian states and all non-Gaussian states of arbitrary dimension at the next level $n=2$, i.e., looking into three phase-space points only. This opens a new possibility for a faithful and efficient test. We show that our foundational approach can constitute two practical tests characterizing nonclassical states reliably and efficiently from a particle and a wave point of views, respectively. It thus makes our method a versatile tool for a wide range of $\mathrm{CV}$ systems in quantum physics and technologies. We illustrate the practical power of our approach by examples. Our proposed approach is fruitful also in other aspects. It provides an experimentally accessible lower bound for nonclassical distance defined via trace norm [42], which is hard to obtain even theoretically. It can also be further extended to identify quantum non-Gaussianity [43-57] under energy constraint.

\section{CRITERIA}

Let us start with a general condition on classicality. A classical state, i.e., a mixture of coherent states, must satisfy

$$
\int d^{2} \alpha P_{\rho_{c}}(\alpha)|f(\alpha)|^{2} \geqslant 0
$$

for an arbitrary $f(\alpha)$ since its Sudarshan-Glauber- $P$ function $P_{\rho_{c}}(\alpha)$ is positive definite $[58,59]$. Our aim is to establish criteria that deal with the Wigner function at discrete phase-space points by choosing $f(\alpha)$ properly. Not only providing a fundamental insight, the Wigner-function approach also leads to two general practical tests broadly applicable for CV systems, as shown later.

To our aim, invoking the convolution between the $P$ function and the $s$-parametrized function [2]

$$
W_{\rho}(\alpha ; s)=\frac{2}{\pi(1-s)} \int d^{2} \beta P_{\rho}(\beta) e^{-\frac{2|\beta-\alpha|^{2}}{1-s}},
$$

we choose $f(\alpha)=\sum_{i=1}^{n} c_{i} e^{-\left|\alpha-\beta_{i}\right|^{2}} \quad(s=0$ for Wigner function, $c_{i}, \beta_{i}$ : arbitrary complex numbers). It yields $\int d^{2} \alpha P_{\rho}(\alpha)|f(\alpha)|^{2}=\sum_{i, j=1}^{n} c_{i}^{*} c_{j} \mathcal{M}_{i j}^{(n)} \geqslant 0$, where

$$
\mathcal{M}_{i j}^{(n)}=\frac{\pi}{2} W_{\rho}\left(\frac{\beta_{i}+\beta_{j}}{2}\right) e^{-\frac{1}{2}\left|\beta_{i}-\beta_{j}\right|^{2}} .
$$

For the classicality to hold for arbitrary $c_{i}$ 's, we deduce the following theorem.

Theorem. An $n \times n$ matrix $\mathcal{M}^{(n)}$ with its elements given by Eq. (3) must be positive semidefinite for a classical state, i.e., $\mathcal{M}^{(n)} \succeq \mathbf{0}$ for all $n \in[1, \infty)$, with arbitrary $\left\{\beta_{1}, \beta_{2}, \ldots \beta_{n}\right\}$.
In other words, we verify nonclassicality if there exists a nonpositive $\mathcal{M}^{(n)} \nsucceq \mathbf{0}$ for any $n$.

\section{HIERARCHY}

By its construction, $\mathcal{M}^{(n+1)} \succeq \mathbf{0}$ implies $\mathcal{M}^{(n)} \succeq \mathbf{0}$ since the matrix $\mathcal{M}^{(n+1)}$ includes $\mathcal{M}^{(n)}$ as its submatrix. That is, there naturally occurs a hierarchy of criteria with $n$ increasing. If nonclassicality is confirmed at the level of $n$, it must be so at the next levels of $n+1$, etc., but the converse is not always true.

Our formulation includes the negativity of Wigner function at the lowest $n=1, \mathcal{M}^{(1)}=\frac{\pi}{2} W(\beta) \nsucceq \mathbf{0}$. Then, it is fundamentally interesting, and practically important, to know how many phase-space points are required to verify noclassicality for states with positive Wigner functions. We prove below that our method can detect nonclassical states comprehensively using only three points $\left\{\beta_{1}, \frac{\beta_{1}+\beta_{2}}{2}, \beta_{2}\right\}$ on a line, i.e., $\mathcal{M}^{(2)} \nsucceq \mathbf{0}$ with

$$
\mathcal{M}^{(2)}=\frac{\pi}{2}\left(\begin{array}{cc}
W\left(\beta_{1}\right) & W\left(\frac{\beta_{1}+\beta_{2}}{2}\right) e^{-\frac{1}{2}\left|\beta_{1}-\beta_{2}\right|^{2}} \\
W\left(\frac{\beta_{1}+\beta_{2}}{2}\right) e^{-\frac{1}{2}\left|\beta_{1}-\beta_{2}\right|^{2}} & W\left(\beta_{2}\right)
\end{array}\right) .
$$

\section{GEOMETRIC INTERPRETATION}

Before demonstrating its usefulness, let us briefly discuss the meaning of the classicality condition $\mathcal{M}^{(2)} \geqslant \mathbf{0}$. One readily finds that all coherent states satisfy $W_{\text {coh }}\left(\frac{\beta_{1}+\beta_{2}}{2}\right) e^{-\frac{1}{2}\left|\beta_{1}-\beta_{2}\right|^{2}}=\sqrt{W_{\text {coh }}\left(\beta_{1}\right) W_{\text {coh }}\left(\beta_{2}\right)}$ yielding $\mathcal{M}^{(2)} \geqslant \mathbf{0}$ for arbitrary $\left\{\beta_{1}, \beta_{2}\right\}$. The linearity of $\mathcal{M}^{(n)}$ with respect to states, $\mathcal{M}_{\sum p_{i} \rho_{i}}^{(2)}=\sum p_{i} \mathcal{M}_{\rho_{i}}^{(2)}$, then makes a general classicality condition $\mathcal{M}^{(2)} \geqslant \mathbf{0}$ for a mixture of coherent states. For a classical state, we thus see that the Wigner function at midpoint $\frac{\beta_{1}+\beta_{2}}{2}$ must be bounded by the geometric mean of the Wigner functions at end points $\beta_{1}$ and $\beta_{2}$, importantly with a scaling factor $e^{-\frac{1}{2}\left|\beta_{1}-\beta_{2}\right|^{2}}$. In fact, this factor results from the commutator $\left[\hat{a}, \hat{a}^{\dagger}\right]=1$ representing the size of vacuum fluctuation.

Gaussian states. Every single-mode Gaussian state can be expressed as a displaced squeezed thermal state

$$
\sigma=\hat{D}(\gamma) \hat{S}(r, \phi) \rho_{t h}(\bar{n}) \hat{S}^{\dagger}(r, \phi) \hat{D}^{\dagger}(\gamma) .
$$

Here $\hat{S}(r, \phi)=\exp \left[-\frac{r}{2}\left(e^{2 i \phi}\left(\hat{a}^{\dagger}\right)^{2}-e^{-2 i \phi} \hat{a}^{2}\right)\right]$ is a squeezing operator with strength $r$ and angle $\phi$ of squeezing axis. $\rho_{t h}(\bar{n})=\sum_{n=0}^{\infty} \frac{\bar{n}^{n}}{(\bar{n}+1)^{n+1}}|n\rangle\langle n|$ is a thermal state with mean number $\bar{n}$. We can readily show $\mathcal{M}^{(2)} \nsucceq \mathbf{0}$ by taking three points along a squeezed axis [Fig. 1(a)], with two end points at a distance $2 d$ and the middle point at the origin. Our test turns out to be successful for a wide range of $d$ as shown in Fig. 1(c). Without loss of generality, we consider an $x$ squeezed thermal state $(\gamma, \phi=0)$, whose Wigner function is given by $W_{\sigma}(q, p)=\frac{2 \mu}{\pi} e^{-2 e^{2\left(r-r_{c}\right)} q^{2}} e^{-2 e^{-2\left(r+r_{c}\right)} p^{2}}$, with purity $\mu=(1+2 \bar{n})^{-1}$ and critical squeezing $r_{c}=-\frac{1}{2} \log \mu$. Section S1 of the Supplemental Material (SM) [60] gives its lowest eigenvalue of $\mathcal{M}^{(2)}$ as

$$
\lambda_{\min , \sigma}=-2 \mu e^{-\left(r-r_{c}\right) \operatorname{coth}\left(r-r_{c}\right)} \sinh \left(r-r_{c}\right)<0,
$$



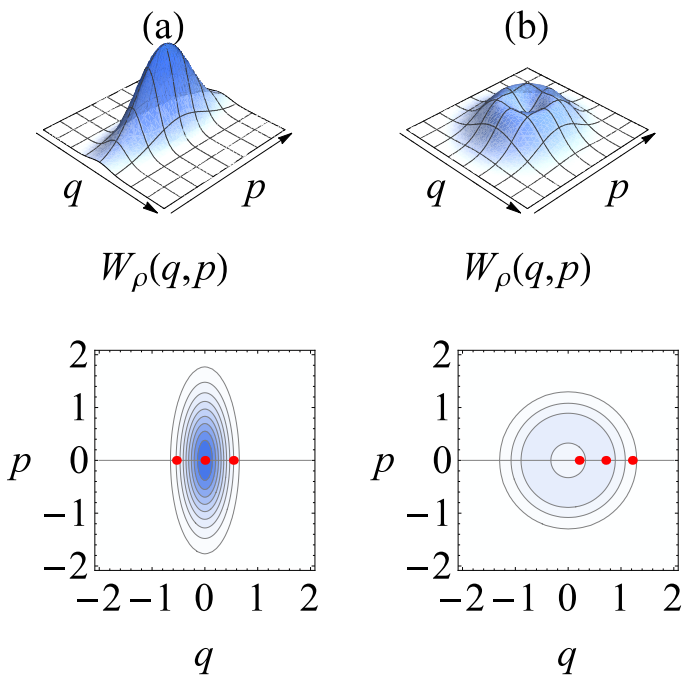

(c)
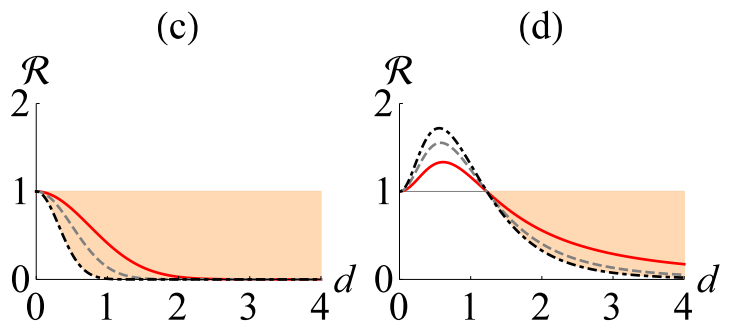

FIG. 1. Wigner function of (a) squeezed vacuum $(r=0.5)$ and (b) a lossy single photon $0.6|0\rangle\langle 0|+0.4| 1\rangle\langle 1|$. Red dots in contour plot represent the phase-space points for $\mathcal{M}^{(2)}$ test. (c) $\mathcal{R}=$ $\frac{W_{\rho}(-d) W_{\rho}(d)}{W_{\rho}(0)^{2} \exp \left(-4 d^{2}\right)}$ for $x$-squeezed states with squeezing $r-r_{c}=0.1$ (red solid) $r-r_{c}=0.2$ (gray dashed) $r-r_{c}=0.4$ (black dot-dashed) and (d) $\mathcal{R}=\frac{W_{\rho}(0) W_{\rho}(2 d)}{W_{\rho}(d)^{2} \exp \left(-4 d^{2}\right)}$ for a Fock state $|n\rangle$ under $80 \%$ loss channel for $n=1$ (red solid), $n=2$ (gray dashed), $n=3$ (black dot-dashed). $\mathcal{R}<1$ (shaded region) confirms nonclassicality for a wide range of displacement $d$.

confirming nonclassicality for every squeezed state $r>r_{c}$, pure or mixed.

Non-Gaussian states. More importantly, the three-points test $\mathcal{M}^{(2)} \nsucceq \mathbf{0}$ can detect a broad range of non-Gaussian states. We first demonstrate its success for all non-Gaussian states of arbitrary truncation in Fock space. This includes as examples all noisy Fock states having positive Wigner functions. In Sec. S3 of SM [60], we further demonstrate that it can be extended to states of practical relevance having infinite Fock-state components.

The Wigner function of an arbitrary Fock-space truncated state (FSTS), $\rho=\sum_{i, j=0}^{N} \rho_{j k}|j\rangle\langle k|$, takes a form $W_{\rho}(\alpha)=$ $\sum_{i, j=0}^{N} \rho_{j k} W_{|j\rangle\langle k|}(\alpha)$, with $W_{|j\rangle\langle k|}(\alpha)$ given in Sec. S2 of SM [60]. As the case of negative Wigner functions is already treated at $n=1$, we focus on the case of positive Wigner functions. Choosing $\beta_{1}=2 d e^{i \varphi}$ and $\beta_{2}=0$ gives $\operatorname{det} \mathcal{M}^{(2)}=$ $\frac{\pi^{2}}{4}\left[W_{\rho}\left(2 d e^{i \varphi}\right) W_{\rho}(0)-W_{\rho}^{2}\left(d e^{i \varphi}\right) e^{-4 d^{2}}\right]$. We thus look into $\mathcal{R}(d)=\frac{W_{\rho}\left(2 d e^{i \varphi}\right) W_{\rho}(0)}{W_{\rho}^{2}\left(d e^{i \varphi}\right) e^{-4 d^{2}}}$ whose value less than 1 verifies nonclassicality. $\mathcal{R}(d)$ is a continuous function of $d$ satisfying $\mathcal{R}(0)=1$. For the FSTS, we always find $\lim _{d \rightarrow \infty} \mathcal{R}(d)=0$ with details in Sec. S2 of SM [60]. Therefore, there must be a finite $d$ satisfying $\mathcal{R}(d)<1$ confirming nonclassicality. Remarkably, it works regardless of $\varphi$, i.e., insensitive to the axis of three points.

As an illustration, in Fig. 1, we plot the ratio $\mathcal{R}$ for (c) squeezed states and (d) Fock states under a 80\%-loss channel. We confirm nonclassicality, $\mathcal{R}<1$, for a broad range of displacement $d$.

\section{NONCLASSICALITY DISTANCE}

It is also a topic of great interest to quantify the degree of nonclassicality for a given state $\rho$. A typical approach is to measure a distance between $\rho$ and its closest classical state $\rho_{c}$ as $\mathcal{N}_{d}(\rho) \equiv \frac{1}{2} \min _{\rho_{c} \in \mathcal{C}}\left\|\rho-\rho_{c}\right\|_{1}$, with $\|\cdot\|_{1}$ the trace norm and $\mathcal{C}$ the set of classical states. This is, however, very hard to obtain even if the state is completely known. Our formalism provides a lower bound for this nonclassical distance $[42,61]$ enabling its practical estimation. With details in Sec. S4 of SM [60], we obtain

$$
\mathcal{N}_{d}(\rho) \geqslant-\frac{\lambda_{\min }}{2 n},
$$

where $\lambda_{\min }$ is the least eigenvalue of $\mathcal{M}^{(n)}$ at the level $n$.

At $n=1$, Eq. (7) shows that a negative value in phase space directly provides a reliable estimate for nonclassical distance. We can further estimate the nonclassical distance of a state with a positive Wigner function by using $\mathcal{M}^{(2)}$. For instance, for a general Gaussian state $\sigma$,

$$
\mathcal{N}_{d}(\sigma) \geqslant \frac{\mu}{2} e^{-\left(r-r_{c}\right) \operatorname{coth}\left(r-r_{c}\right)} \sinh \left(r-r_{c}\right),
$$

using Eq. (6), which is beyond the results in Refs. [42,61] addressing only pure Gaussian states. We also establish connection between our approach and nonclassical depth [62] in S9 of SM [60].

\section{QNG}

Furthermore, our formalism also leads to a criterion on quantum non-Gaussianity (QNG) manifesting that a state cannot be a mixture of Gaussian states. $[40,43-50,54,56]$. QNG has recently attracted much attention in CV quantum informatics as there exist numerous quantum tasks essentially requiring it beyond Gaussian resources, e.g., quantum computation [63,64], entanglement distillation [65-67], and error correction [68].

With details in Sec. S5 of SM [60], if the least eigenvalue of $\mathcal{M}_{\rho}^{(2)}$ for a state $\rho$ with energy $E$ satisfies

$$
\lambda_{\min }<\mathcal{B}(E) \equiv-\frac{2 \sqrt{E}}{(\sqrt{E+1}+\sqrt{E})^{\sqrt{1+E^{-1}}}} .
$$

it confirms QNG. In Fig. 2, we plot $\Delta \lambda_{\min }=\mathcal{B}(E)-\lambda_{\min }$ for a non-Gaussian state $\rho=\hat{S}(r)\{f|2\rangle\langle 2|+(1-f)| 0\rangle\langle 0|\} \hat{S}^{\dagger}(r)$ with squeezing $\hat{S}(r)=e^{\frac{r}{2}\left(a^{\dagger}\right)^{2}-\frac{r}{2} a^{2}}$. As seen from Fig. 2(a), our criterion detects QNG with $f<\frac{1}{2}$ (positive Wigner function) for a squeezing $r \gtrsim 0.237$. Note that the squeezing operation does not create QNG as it is a Gaussian operation. In this context, the result also represents the QNG of $f|2\rangle\langle 2|+(1-f)| 0\rangle\langle 0|$ without squeezing. A recent ion-trap experiment realized a measurement in squeezed Fock basis, 

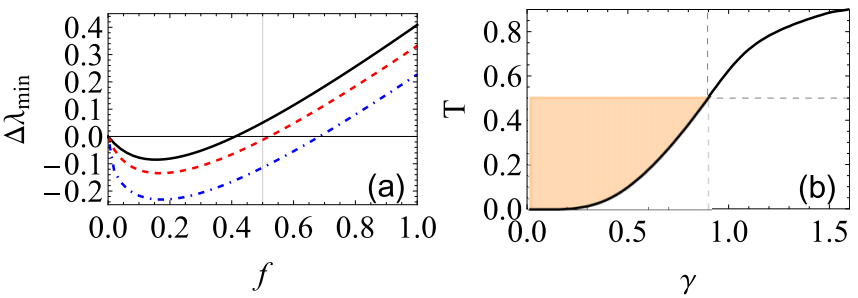

FIG. 2. (a) $\Delta \lambda_{\min }=\mathcal{B}(E)-\lambda_{\min }$ for the state $\hat{S}(r)\{f|2\rangle\langle 2|+$ $(1-f)|0\rangle\langle 0|\} \hat{S}^{\dagger}(r)$ with $r=0$ (blue dot-dashed), $r=0.2$ (red dashed) and $r=0.5$ (black solid). The QNG-detectable region, $\Delta \lambda_{\min }>0$, broadens with squeezing $r$. (b) The QNG of a fourcomponent cat state $|\mathrm{C}\rangle \sim|\gamma\rangle+\left|\gamma \mathrm{e}^{\mathrm{i} \frac{\pi}{2}}\right\rangle+\left|\gamma \mathrm{e}^{\mathrm{i} \pi}\right\rangle+\left|\gamma \mathrm{e}^{\mathrm{i} \frac{3 \pi}{2}}\right\rangle[70]$ is confirmed under a loss channel ( $T$ : transmittance) for each $\gamma$. Black solid represents the minimum $T$ above which QNG is verified by Eq. (9). The yellow region represents the case of positive Wigner function.

$\{\hat{S}(r)|n\rangle: n=0,1, \cdots\}$ [69]. This can be adopted to verify QNG of states $\hat{S}(r) \rho_{\mathrm{nG}} \hat{S}^{\dagger}(r)$ without performing squeezing on a non-Gaussian state $\rho_{\mathrm{nG}}$ enhancing the range of QNG detection. Fig. 2(b) gives another example of a positive Wigner function with its QNG verified.

\section{PRACTICAL TESTS}

The Wigner function corresponds to the number parity after displacement, i.e., $W_{\rho}(\alpha)=\frac{2}{\pi} \operatorname{tr}\left[\hat{D}^{\dagger}(\alpha) \rho \hat{D}(\alpha)(-1)^{\hat{n}}\right]$. It is routinely measured in various systems, e.g., ion-trap [40,71] and circuit-QED [72], for which our proposed test $\mathcal{M}^{(2)}$ can thus directly characterize nonclassicality. On the other hand, we can also derive alternative, practical, schemes out of Wigner-function framework, which can test nonclassicality reliably and efficiently against experimental imperfections. First, we present a generalized formalism to use on-off detectors registering photons without photon-number resolving (PNR). Second, we also present a marginal version of Wignerfunction test, i.e., using $M(q)=\int d p W_{\rho}(q, p)$, which can be measured by homodyne detection well established for a wide variety of quantum systems including quantum optics [29], trapped ion [73], atomic ensemble [74], circuit cavity QED $[75,76]$, and optomechanics [77,78]. Both of our proposed tests are powerful against noise with wide applicability.

On-off detector array. When an input light is equally divided via beam splitting to impinge on $N$ on-off detectors, the probability of $k$ detectors clicking is [79]

$$
p_{k}[\rho]=\operatorname{tr}\left[\rho: \frac{N}{k !(N-k) !}\left(e^{-\frac{\eta \hat{n}}{N}}\right)^{N-k}\left(1-e^{-\frac{\eta \hat{n}}{N}}\right)^{k}:\right],
$$

with $\eta$ detector efficiency and : $\hat{O}:$ normal-ordering. The counting statistics $p_{k}$ above can also be obtained via the time-multiplexing approach using a single detector [33]. We first generalize our criterion to $s$-parametrized function $W_{\rho}(\alpha ; s)$ [2] to use the counting statistics $p_{k}[\rho]$ from on-off detectors. Choosing $f(\alpha)=\sum_{i=1}^{n} c_{i} e^{-\frac{\left|\alpha-\beta_{i}\right|^{2}}{1-s}}$ in Eq. (1) with the convolution in Eq. (2), we obtain $\int d^{2} \alpha P_{\rho}(\alpha)|f(\alpha)|^{2}=$

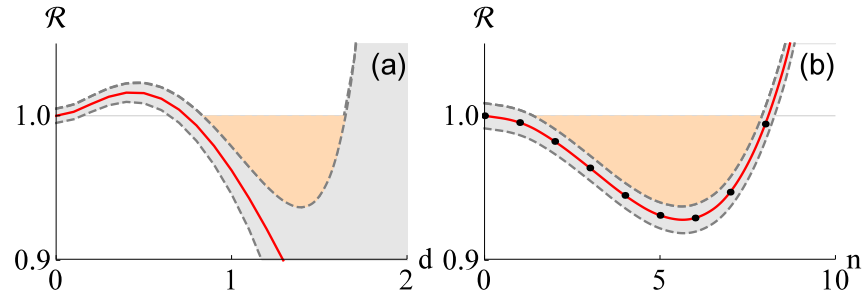

FIG. 3. (a) Testing a Fock state $|n=2\rangle$ under $50 \%$ loss channel mixed with a thermal photon $\bar{n}=0.05$ using $N=1$ on-off detectors. $\mathcal{R}=\frac{W_{\rho}\left(d_{1} ; s\right) W_{\rho}\left(d_{1}+2 d ; s\right)}{W_{\rho}\left(d_{1}+d ; s\right)^{2} \exp \left(-4 d^{2}\right)}(s=-1.86$, red solid $)$ against displacement $d$ with $d_{1}=1$. (b) Homodyne test for a phase-diffused squeezed state with $\mathcal{R}=\frac{M_{\rho}^{\sigma}[-n] M_{\rho}^{\sigma}[n]}{M_{\rho}^{\sigma}[0]^{2} \exp \left(-4 n^{2} \sigma^{2}\right)}$ (red solid) against $n$ with binning size $\sigma=$ 0.1 . $n$ : bin number for quadrature $q=n \sigma$. Grey shades represent the size of error due to finite data (a) $\sim 10^{5}$ and (b) $\sim 10^{6}$.

$\sum_{i, j=1}^{n} c_{i}^{*} c_{j} \mathcal{M}_{i j}^{(s, n)} \geqslant 0$, where

$$
\mathcal{M}_{i j}^{(s)}=\frac{\pi(1-s)}{2} W_{\rho}\left(\frac{\beta_{i}+\beta_{j}}{2} ; s\right) e^{-\frac{1}{1-s} \frac{\left|\beta_{i}-\beta_{j}\right|^{2}}{2}} .
$$

Our classicality condition is readily generalized to $\mathcal{M}^{(s, n)} \succeq \mathbf{0}$ for an arbitrary $s$ using elements in Eq. (11).

We find the connection between the $s$-parametrized functions and the counting statistics $p_{k}$ in S6 of SM $[60]$ as

$$
\left(\begin{array}{c}
W_{\rho}\left(\alpha ; s_{0}\right) \\
W_{\rho}\left(\alpha ; s_{1}\right) \\
\cdots \\
W_{\rho}\left(\alpha ; s_{N-1}\right) \\
1
\end{array}\right)=T^{-1}\left(\begin{array}{c}
p_{0} \\
p_{1} \\
\cdots \\
p_{N-1} \\
p_{N}
\end{array}\right),
$$

with each $s_{m}=1-\frac{2 N}{(N-m) \eta} \quad(m=0, \cdots, N-1)$. Equation (12) means that $N$ different $s$-parametrized distributions $W_{\rho}\left(\alpha ; s_{m}\right)$ are determined by the counting statistics $\left\{p_{0}, \cdots, p_{N}\right\}$ obtained for a displaced state $\hat{D}^{\dagger}(\alpha) \rho \hat{D}(\alpha)$. Furthermore, we prove in Sec. S2 of SM [60] that $\mathcal{M}^{(s, n=2)}$ (three points test) can detect all nonclassical Gaussian and non-Gaussian states (FSTSs), importantly for an arbitrary s. This broader applicability beyond Wigner function makes our test robust against noise.

Let us illustrate the case of testing a Fock state $|n\rangle$ under $50 \%$ loss channel mixed with a thermal photon $\bar{n}=0.05$ by using only $N=1$ on-off detector of efficiency $\eta=0.7$ [80]. We further consider an error due to finite data acquisition $\sim 10^{5}$ (Sec. S8 of SM [60]). Our 3-points test adopting $W_{\rho}(\alpha ; s=-1.86)$ is accomplished with $s_{m}=1-\frac{2 N}{(N-m) \eta}$ for $m=0$. As shown in Fig. 3(a), there exists a range of displacement to detect nonclassicality substantially beating the error. For instance, we have the signal to noise ratio as $\frac{1-\mathcal{R}}{\Delta \mathcal{R}}=2.48$ at $d=1.1$. We also demonstrate the successful detection for other noisy Fock states with error analysis in Sec. S8 of SM [60].

Homodyne test. We next present a test using a marginal distribution $M(q)=\int d p W_{\rho}(q, p)$. Homodyne detection to measure $M(q)$ is highly efficient, but requires a careful analysis. It is because that the actual homodyne data is coarsegrained due to finite binning, which may lead to a false detection of nonclassical effects [81-83]. Let $\sigma$ be the binning 
size of homodyne data. Then all data in the range $[-\sigma / 2, \sigma / 2]$ belong to the same bin yielding a coarse-grained distribution $M_{\rho}^{\sigma}[n] \equiv \int_{-\sigma / 2}^{\sigma / 2} d \delta M_{\rho}(n \sigma+\delta)$ ( $n$ : bin number representing mean quadrature $q=n \sigma)$. A classicality condition $\mathcal{M}^{(H)} \geqslant 0$ then emerges with its elements

$$
\mathcal{M}_{i j}^{(H)} \equiv \frac{\pi}{2} M_{\rho}^{\sigma}\left[m_{i}+m_{j}+k\right] e^{-2\left(m_{i}-m_{j}\right)^{2} \sigma^{2}},
$$

where $k$ can be either 0 or 1 , with details in Sec. S7 of SM [60].

We prove in SM [60] that this marginal test even with a coarse-grained information detects all nonclassical Gaussian and non-Gaussian states (FSTSs). In Fig. 3(b), we show the result for a squeezed state $(r=0.3)$ under phase diffusion, $\mathcal{D}[\rho]=\int d \phi \sqrt{\frac{1}{2 \pi \Delta^{2}}} e^{-\frac{\phi^{2}}{2 \Delta^{2}}} e^{i \hat{n} \phi} \rho e^{-i \hat{n} \phi}$ leaving no squeezing at $\Delta=1.2$. Our homodyne test under coarse-graining $(\sigma=0.1)$ clearly manifests nonclassicality over 7 standard deviation, $\frac{1-\mathcal{R}}{\Delta \mathcal{R}}=7.11$. We also illustrate other cases in Sec. S8 of $\mathrm{SM}[60]$.

\section{CONCLUSION}

A phase-space approach usually provides us with a valuable insight into quantum physics [84]. While negativity is one manifestation of nonclassicality, recent studies made it clear that all nonclassical states even without negativity are valuable resources for quantum information science [8-11,18]. It is thus critically important to establish a comprehensive framework of addressing nonclassical states with and without negativity covering a wide range of quantum systems. We have introduced a hierarchy of nonclassicality conditions that can address nonclassicality beyond negativity effectively and efficiently. Our approach makes it possible to analyze all nonclassical Gaussian states and non-Gaussian states using three phase-space points. Our formalism further provides a lower bound for nonclassical distance and a criterion to detect quantum non-Gaussianity with positive Wigner functions.
Remarkably, our foundational approach also constitutes two practical tests looking into particle nature (number parity) and wave nature (marginal distribution), making a versatile tool for CV systems broadly. We illustrated the practical power of our tests adopting nonideal on-off detectors without resolving photon numbers and coarse-grained homodyne detection, respectively.

We hope our paper could further stimulate works related to nonclassical effects from both a fundamental and a practical perspective. Our approach here clearly indicates that the information on nonclassicality is sufficiently imbedded in phase space even at a few points. Our geometric interpretation on classicality has stipulated the relation among the values of Wigner function, which is fundamentally associated with quantum fluctuation represented by a commutation relation or uncertainty principle. This seems worthwhile to further purse in studying nonclassicality for quantum multipartite systems as well. In the near term, we anticipate our framework can be useful for both theoretical and experimental analysis of quantum systems. In particular, our proposed tests can address all different CV systems including quantum optics, nano- or optomechanics, atomic ensemble, and circuit cavity QED, and so on.

Note added. We became aware of a closely related work by Bohmann, Agudelo and Sperling [85]. We note that our main idea and some results were earlier presented at an international conference [86].

\section{ACKNOWLEDGMENTS}

J.P. acknowledges support by the National Research Foundation of Korea (NRF) Grant funded by the Korea government (MSIT) (No. NRF-2019R1G1A1002337). J.L. is supported by a KIAS Individual Grant (No. CG073101) at Korea Institute for Advanced Study. H.N. is supported by a grant NPRP13S-0205-200258 from Qatar National Research Fund.
[1] E. Wigner, On the quantum correction for thermodynamic equilibrium, Phys. Rev. 40, 749 (1932).

[2] S. M. Barnett and P. M. Radmore, Methods in Theoretical Quantum Optics (Oxford University Press, New York, 2003).

[3] S. L. Braunstein and P. Van Loock, Quantum information with continuous variables, Rev. Mod. Phys. 77, 513 (2005).

[4] C. Weedbrook, S. Pirandola, R. García-Patrón, N. J. Cerf, T. C. Ralph, J. H. Shapiro, and S. Lloyd, Gaussian quantum information, Rev. Mod. Phys. 84, 621 (2012).

[5] J. Weinbub and D. K. Ferry, Recent advances in Wigner function approaches, Appl. Phys. Rev. 5, 041104 (2018).

[6] E. Knill, R. Laflamme, and G. J. Milburn, A scheme for efficient quantum computation with linear optics, Nature (London) 409, 46 (2001).

[7] P. Kok, W. J. Munro, K. Nemoto, T. C. Ralph, J. P. Dowling, and G. J. Milburn, Linear optical quantum computing with photonic qubits, Rev. Mod. Phys. 79, 135 (2007).

[8] M. S. Kim, W. Son, V. Buzek, and P. L. Knight, Entanglement by a beam splitter: Nonclassicality as a prerequisite for entanglement, Phys. Rev. A 65, 032323 (2002).
[9] W. Xiang-bin, Theorem for the beam-splitter entangler, Phys. Rev. A 66, 024303 (2002).

[10] J. K. Asbóth, J. Calsamiglia, and H. Ritsch, Computable Measure of Nonclassicality for Light, Phys. Rev. Lett. 94, 173602 (2005).

[11] H. Nha and M. S. Zubairy, Uncertainty Inequalities as Entanglement Criteria for Negative Partial-Transpose States, Phys. Rev. Lett. 101, 130402 (2008).

[12] R. Tahira, M. Ikram, H. Nha, and M. S. Zubairy, Entanglement of Gaussian states using a beam splitter, Phys. Rev. A 79, 023816 (2009).

[13] B. Yadin, F. C. Binder, J. Thompson, V. Narasimhachar, M. Gu, and M. S. Kim, Operational Resource Theory of Continuous-Variable Nonclassicality, Phys. Rev. X 8, 041038 (2018).

[14] H. Kwon, K. C. Tan, T. Volkoff, and H. Jeong, Nonclassicality as a Quantifiable Resource for Quantum Metrology, Phys. Rev. Lett. 122, 040503 (2019).

[15] K. C. Tan and H. Jeong, Resource theories of nonclassical light, Quantum Rep. 1, 151 (2019). 
[16] H. Pashayan, J. J. Wallman, and S. D. Bartlett, Estimating Outcome Probabilities of Quantum Circuits Using Quasiprobabilities, Phys. Rev. Lett. 115, 070501 (2015).

[17] S. Rahimi-Keshari, A. P. Lund, and T. C. Ralph, What Can Quantum Optics Say about Computational Complexity Theory?, Phys. Rev. Lett. 114, 060501 (2015).

[18] R. Takagi, B. Regula, K. Bu, Z.-W. Liu, and G. Adesso, Operational Advantage of Quantum Resources in Subchannel Discrimination, Phys. Rev. Lett. 122, 140402 (2019).

[19] B. Regula, L. Lami, G. Ferrari, and R. Takagi, Operational Quantification of Continuous-Variable Quantum Resources, Phys. Rev. Lett. 126, 110403 (2021).

[20] G. S. Agarwal and K. Tara, Nonclassical character of states exhibiting no squeezing or sub-Poissonian statistics, Phys. Rev. A 46, 485 (1992).

[21] D. N. Klyshko, Observable signs of nonclassical light, Phys. Lett. A 213, 7 (1996)

[22] B. Arvind and N. Mukunda, Non-classical photon statistics for two-mode optical fields, J. Phys. A 29, 5855 (1996); B. Arvind, N. Mukunda, and R. Simon, Characterizations of classical and nonclassical states of quantized radiation, ibid. 31, 565 (1998).

[23] R. Simon, M. Selvadoray, B. Arvind, and N. Mukunda, Necessary and sufficient classicality conditions on photon number distributions, arXiv:quant-ph/9709030.

[24] C. T. Lee, General criteria for nonclassical photon statistics in multimode radiations, Opt. Lett. 15, 1386 (1990).

[25] J. S. Ivan, S. Haturvedi, E. Ercolessi, G. Marmo, G. Morandi, N. Mukunda, and R. Simon, Entanglement and nonclassicality for multimode radiation-field states, Phys. Rev. A 83, 032118 (2011).

[26] B. Hage, A. Franzen, J. DiGuglielmo, P. Marek, J. Fiurásek, and R. Schnabel, On the distillation and purification of phasediffused squeezed states, New J. Phys. 9, 227 (2007).

[27] R. Filip, Distillation of quantum squeezing, Phys. Rev. A 88, 063837 (2013).

[28] R. Filip, Squeezed-state generation from single-photon sources, Phys. Rev. A 90, 043854 (2014).

[29] A. I. Lvovsky and M. G. Raymer, Continuous-variable optical quantum-state tomography, Rev. Mod. Phys. 81, 299 (2009).

[30] T. Kiesel and W. Vogel, Nonclassicality filters and quasiprobabilities, Phys. Rev. A 82, 032107 (2010).

[31] T. Kiesel, W. Vogel, B. Hage, and R. Schnabel, Direct Sampling of Negative Quasiprobabilities of a Squeezed State, Phys. Rev. Lett. 107, 113604 (2011).

[32] A. Luis, J. Sperling, and W. Vogel, Nonclassicality Phase-Space Functions: More Insight with Fewer Detectectors, Phys. Rev. Lett. 114, 103602 (2015).

[33] M. Bohmann, J. Tiedau, T. Bartley, J. Sperling, C. Silberhorn, and W. Vogel, Incomplete Detection of Nonclassical PhaseSpace Distributions, Phys. Rev. Lett. 120, 063607 (2018).

[34] M. Bohmann and E. Agudelo, Phase-Space Inequalities Beyond Negativities, Phys. Rev. Lett. 124, 133601 (2020).

[35] K. Banaszek and K. Wódkiewicz, Testing Quantum Nonlocality in Phase Space, Phys. Rev. Lett. 82, 2009 (1999).

[36] S.-W. Lee, H. Jeong, and D. Jaksch, Testing quantum nonlocality by generalized quasiprobability functions, Phys. Rev. A 80, 022104 (2009)

[37] S.-W. Lee, M. Paternostro, J. Lee, and H. Jeong, Testing genuine multipartite nonlocality in phase space, Phys. Rev. A 87, 022123 (2013).
[38] G. Adesso and S. Piano, Theory of Genuine Tripartite Nonlocality of Gaussian States, Phys. Rev. Lett. 112, 010401 (2014).

[39] B. Xu, T. Tufarelli, and G. Adesso, Genuine multipartite nonlocality of permutationally invariant Gaussian states, Phys. Rev. A 95, 012124 (2017).

[40] J. Park, J. Zhang, J. Lee, S.-W. Ji, M. Um, D. Lv, K. Kim, and H. Nha, Testing Nonclassicality and Non-Gaussianity in Phase Space, Phys. Rev. Lett. 114, 190402 (2015).

[41] J. Park and H. Nha, Demonstrating nonclassicality and nonGaussianity of single-mode fields: Bell-type tests using generalized phase-space distributions, Phys. Rev. A 92, 062134 (2015).

[42] M. Hillery, Nonclassical distance in quantum optics, Phys. Rev. A 35, 725 (1987).

[43] R. Filip and L. Mišta, Jr., Detecting Quantum States with a Positive Wigner Function beyond Mixtures of Gaussian States, Phys. Rev. Lett. 106, 200401 (2011).

[44] M. Ježek, I. Straka, M. Mičuda, M. Dušek, J. Fiurášek, and R. Filip, Experimental Test of the Quantum Non-Gaussian Character of a Heralded Single-Photon State, Phys. Rev. Lett. 107, 213602 (2011)

[45] I. Straka, A. Predojević, T. Huber, L. Lachman, L. Butschek, M. Miková, M. Mičuda, G. S. Solomon, G. Weihs, M. Ježek, and R. Filip, Quantum non-Gaussian Depth of Single-Photon States, Phys. Rev. Lett. 113, 223603 (2014).

[46] I. Straka, L. Lachman, J. Hloušek, M. Miková, M. Mičuda, M. Ježek, and R. Filip, Quantum non-Gaussian multiphoton light, npj Quantum Inf. 4, 4 (2018).

[47] L. Lachman, I. Straka, J. Hloušek, M. Ježek, and R. Filip, Faithful Hierarchy of Genuine $n$-Photon Quantum Non-Gaussian Light, Phys. Rev. Lett. 123, 043601 (2019).

[48] M. G. Genoni, M. L. Palma, T. Tufarelli, S. Olivares, M. S. Kim, and M. G. A. Paris, Detecting quantum non-Gaussianity via the Wigner function, Phys. Rev. A 87, 062104 (2013).

[49] C. Hughes, M. G. Genoni, T. Tufarelli, M. G. A. Paris, and M. S. Kim, Quantum non-Gaussianity witnesses in phase space, Phys. Rev. A 90, 013810 (2014).

[50] L. Happ, M. Efremov, H. Nha, and W. P. Schleich, Sufficient condition for a quantum state to be genuinely quantum nonGaussian, New J. Phys. 20, 023046 (2018).

[51] B. Kühn and W. Vogel, Quantum non-Gaussianity and quantification of nonclassicality, Phys. Rev. A 97, 053823 (2018).

[52] R. Takagi and Q. Zhuang, Convex resource theory of nonGaussianity, Phys. Rev. A 97, 062337 (2018).

[53] F. Albarelli, M. G. Genoni, M. G. A. Paris, and A. Ferraro, Resource theory of quantum non-Gaussianity and Wigner negativity, Phys. Rev. A 98, 052350 (2018).

[54] J. Park, J. Lee, K. Baek, S.-W. Ji, and H. Nha, Faithful measure of quantum non-Gaussianity via quantum relative entropy, Phys. Rev. A 100, 012333 (2019).

[55] J. Park, J. Lee, and H. Nha, Entropic nonclassicality and quantum non-Gaussianity tests via beam splitting, Sci. Rep. 9, 17835 (2019)

[56] J. Lee, J. Park, and H. Nha, Quantum non-Gaussianity and secure quantum communication, npj Quantum Inf. 5, 49 (2019).

[57] J. Park et al., Revealing nonclassicality beyond Gaussian states via a single marginal distribution, Proc. Nat. Acad. Sci. USA 114, 891 (2017).

[58] E. C. G. Sudarshan, Equivalence of Semiclassical and Quantum Mechanical Descriptions of Statistical Light Beams, Phys. Rev. Lett. 10, 277 (1963). 
[59] R. J. Glauber, Coherent and Incoherent States of the Radiation Field, Phys. Rev. 131, 2766 (1963).

[60] See Supplemental Material at http://link.aps.org/supplemental/ 10.1103/PhysRevResearch.3.043116 for details on deriving an optimal test for a Gaussian state, proving that the tests via sparametrized functions can detect all nonclassical Gaussian and non-Gaussian states of arbitrary dimension, showing that our test can be extended to infinite-dimensional states, deriving a criterion for quantum non-Gaussianity, deriving the connection between s-parametrized function and on-off counting statistics, proving that a marginal distribution can detect all Gaussian and non-Gaussian states and demonstrating the practical power of our tests against experimental imperfections with numerous examples.

[61] R. Nair, Nonclassical distance in multimode bosonic systems, Phys. Rev. A 95, 063835 (2017).

[62] C. T. Lee, Measure of the nonclassicality of nonclassical states, Phys. Rev. A 44, R2775 (1991).

[63] S. Lloyd and S. L. Braunstein, Quantum Computation over Continuous Variables, Phys. Rev. Lett. 82, 1784 (1999).

[64] N. C. Menicucci, P. van Loock, M. Gu, C. Weedbrook, T. C. Ralph, and M. A. Nielsen, Universal Quantum Computation with Continuous-Variable Cluster States, Phys. Rev. Lett. 97, 110501 (2006).

[65] J. Eisert and M. B. Plenio, Conditions for the Local Manipulation of Gaussian States, Phys. Rev. Lett. 89, 097901 (2002).

[66] J. Fiurášek, Gaussian Transformations and Distillation of Entangled Gaussian States, Phys. Rev. Lett. 89, 137904 (2002).

[67] G. Giedke and J. I. Cirac, Characterization of Gaussian operations and distillation of Gaussian states, Phys. Rev. A 66, 032316 (2002)

[68] J. Niset, J. Fiurášek, and N. J. Cerf, No-Go Theorem for Gaussian Quantum Error Correction, Phys. Rev. Lett. 102, 120501 (2009).

[69] D. Kienzler, C. Flühmann, V. Negnevitsky, H.-Y. Lo, M. Marinelli, D. Nadlinger, and J. P. Home, Observation of Quantum Interference between Separated Mechanical Oscillator Wave Packets, Phys. Rev. Lett. 116, 140402 (2016).

[70] S. Puri, A. Grimm, P. Campagne-Ibarcq, A. Eickbusch, K. Noh, G. Roberts, L. Jiang, M. Mirrahimi, M. H. Devoret, and S. M. Girvin, Stabilized Cat in a Driven Nonlinear Cavity: A FaultTolerant Error Syndrome Detector, Phys. Rev. X 9, 041009 (2019).

[71] D. Leibfried, D. M. Meekhof, B. E. King, C. Monroe, W. M. Itano, and D. J. Wineland, Experimental Determination of the Motional Quantum State of a Trapped Atom, Phys. Rev. Lett. 77, 4281 (1996).

[72] L. Sun, A. Petrenko, Z. Leghtas, B. Vlastakis, G. Kirchmair, K. M. Sliwa, A. Narla, M. Hatridge, S. Shankar, J. Blumoff et al., Tracking photon jumps with repeated quantum nondemolition parity measurements, Nature (London) 511, 444 (2014).

[73] R. Gerritsma et al., Quantum Simulation of the Klein Paradox with Trapped Ions, Phys. Rev. Lett. 106, 060503 (2011).

[74] T. Fernholz, H. Krauter, K. Jensen, J. F. Sherson, A. S. Sørensen, and E. S. Polzik, Spin Squeezing of Atomic Ensembles via Nuclear-Electronic Spin Entanglement, Phys. Rev. Lett. 101, 073601 (2008).

[75] F. Mallet, M. A. Castellanos-Beltran, H. S. Ku, S. Glancy, E. Knill, K. D. Irwin, G. C. Hilton, L. R. Vale, and K. W. Lehnert, Quantum State Tomography of an Itinerant Squeezed Microwave Field, Phys. Rev. Lett. 106, 220502 (2011).

[76] C. Eichler, D. Bozyigit, C. Lang, L. Steffen, J. Fink, and A. Wallraff, Experimental State Tomography of Itinerant Single Microwave Photons, Phys. Rev. Lett. 106, 220503 (2011).

[77] J. B. Hertzberg, T. Rocheleau, T. Ndukum, M. Savva, A. A. Clerk, and K. C. Schwab, Back-action-evading measurements of nanomechanical motion, Nat. Phys. 6, 213 (2010).

[78] M. R. Vanner, J. Hofer, G. D. Cole, and M. Aspelmeyer, Cooling-by-measurement and mechanical state tomography via pulsed optomechanics, Nat. Commun. 4, 2295 (2013).

[79] J. Sperling, W. Vogel, and G. S. Agarwal, True photocounting statistics of multiple on-off detectors, Phys. Rev. A 85, 023820 (2012).

[80] A. E. Lita, A. J. Miller, and S. W. Nam, Counting near-infrared single-photons with 95\% efficiency, Opt. Express 16, 3032 (2008).

[81] J. Schneeloch, P. B. Dixon, G. A. Howland, C. J. Broadbent, and J. C. Howell, Violation of Continuous-Variable EinsteinPodolsky-Rosen Steering with Discrete Measurements, Phys. Rev. Lett. 110, 130407 (2013).

[82] D. S. Tasca, Ł. Rudnicki, R. M. Gomes, F. Toscano, and S. P. Walborn, Reliable Entanglement Detection under CoarseGrained Measurements, Phys. Rev. Lett. 110, 210502 (2013).

[83] J. Park, S.-W. Ji, J. Lee, and H. Nha, Gaussian states under coarse-grained continuous variable measurements, Phys. Rev. A 89, 042102 (2014).

[84] S. De Bievre, D. B. Horoshko, G. Patera, and M. I. Kolobov, Measuring Nonclassicality of Bosonic Field Quantum States via Operator Ordering Sensitivity, Phys. Rev. Lett. 122, 080402 (2019).

[85] M. Bohmann, E. Agudelo, and J. Sperling, Probing nonclassicality with matrices of phase-space distributions, Quantum 4, 343 (2020).

[86] J. Park and H. Nha, Efficient and faithful criteria on nonclassicality for continuous variables, 15th International Conference on Squeezed States and Uncertainty Relations, Jeju, Korea, ICSSUR2017 (2017). 